\title{
FEBUXOSTAT MODULATES OXIDATIVE AND APOPTOTIC PATHWAYS IN ACUTE DOXORUBICIN-INDUCED CARDIOTOXICITY: AN EXPERIMENTAL ANIMAL MODEL STUDY
}

\author{
HAYDER M AL-KURAISHY ${ }^{1}$, ALI I AL-GAREEB ${ }^{2}$, HANY AKEEL NAJI ${ }^{3}$
}

${ }^{1}$ Department of Clinical Pharmacology, Medicine and Therapeutic, Medical Faculty College of Medicine, Al-Mustansiriya University, Baghdad, Iraq. ${ }^{2}$ Department of Clinical Pharmacology, Medicine and Therapeutic, Medical Faculty College of Medicine, Al-Mustansiriya University, Baghdad, Iraq. ${ }^{3}$ Department of Pharmacology . Email: hayderm36@yahoo.com

Received: 21 December 2018, Revised and Accepted: 31 January 2019

ABSTRACT

Objectives: Doxorubicin is one of the most important and powerful anticancer drugs, the most pronounced limitation for its use is toxicity on normal cells. Mechanism of doxorubicin-induced cardiotoxicity (DIC) is multifactorial and complex, including direct DNA damage, formation of free radicals, interference with DNA repair, and activation of immune reactions. Febuxostat is a non-purine-selective xanthine oxidase inhibitor decrease the production of uric acid. The aim of the present study was to evaluate the influence of febuxostat on doxorubicin-induced acute cardiotoxicity in rats regarding oxidative stress and antiapoptotic effects.

Methods: A total of 30 Sprague Dawley male rats were used which subdivided into three groups: Group I (negative control group) received normal saline for 10 days, Group II (positive control group) received normal saline plus single dose of doxorubicin (15 mg/kg, IP), and Group III (treated group) received febuxostat (10 mg/kg, po), for 10 successive days plus single dose of doxorubicin (15 mg/kg, I.P.). Serum brain natriuretic peptide (BNP), cardiac troponin I (cTn-I), caspase-3, glutathione peroxidase (GSH-Px), lipid peroxidase (LPO), malondialdehyde (MDA), and tumor necrosis factor alpha were estimated by ELISA kit method.

Results: Febuxostat administration before doxorubicin led to significant decrease on cardiac troponin, caspase-3, and elevation in GSH-Px levels significantly $\mathrm{p}<0.05$. While the effects of febuxostat on BNP, LPO, MDA, tumor necrosis-alpha were insignificant $\mathrm{p}>0.05$ compare to doxorubicin.

Conclusion: Febuxostat attenuates DIC through modulation of antioxidant, anti-inflammatory, and antiapoptotic biomarkers.

Keywords: Doxorubicin, Febuxostat, Cardiotoxicity.

(C) 2019 The Authors. Published by Innovare Academic Sciences Pvt Ltd. This is an open access article under the CC BY license (http://creativecommons. org/licenses/by/4. 0/) DOI: http://dx.doi.org/10.22159/ajpcr.2019.v12i4.31162

\section{INTRODUCTION}

Doxorubicin is one of the most important and prominent anticancer drugs. Doxorubicin has the ability to fight rapid dividing cells and to slow the progression of the many malignant diseases [1]. However, clinical usefulness of doxorubicin is limited by its cumulative, dosedependent progressive cardiotoxicity [2].

It has been categorized three distinct types of doxorubicin-induced cardiotoxicity (DIC), including acute (within days), early-onset chronic (within weeks), and chronic progressive cardiotoxicity (within monthsyears) after drug administration. Acute DIC may occur during doxorubicin initiation or immediately later which appears as temporary ECG abnormalities, while chronic DIC is presented as congestive heart failure [3].

Different molecular mechanisms are involved in DIC which is multifactorial and complex, include direct DNA damage, formation of free reactive oxygen radicals, interference with DNA repair, alterations of cellular calcium homeostasis, mitochondria injury, lysosomal changes, and apoptosis [4,5]. The heart is particularly sensitive to the oxidative damage due to low levels of antioxidant enzymes, large density of mitochondrial, and high rate of oxygen consumption [6]. Moreover, repeated doxorubicin administration promotes nitric oxide (NO) production in the myocardium through induction of inducible NO synthase expression. The cardiotoxic effect of NO is due to the formation of reactive nitrogen species which attack cellular biomolecules, causing energy imbalance and death [7].

Febuxostat is a non-purine-selective xanthine oxidase inhibitor, blocks the active site of xanthine oxidase. Hence, febuxostat decreases the production of uric acid [8]. Krishnamurthy et al. study demonstrated a significant effect of febuxostat on attenuation of DIC through reduction of cardiac injury biomarkers and activation of endogenous antioxidant capacity as febuxostat improves diastolic pressure with significant positive inotropic and lusitropic effects [9]. These findings give a clue that febuxostat could produce a cardioprotective due to its antioxidant effect.

Therefore, the aim of the present study was to evaluate the influence of febuxostat on doxorubicin-induced acute cardiotoxicity in rats regarding oxidative stress and antiapoptotic effects.

\section{METHODS}

A total of 30 Sprague Dawley male rats were used in this study, which purchased from International Center for Cancer and Medical Genetic Researches. Rat's body weight ranged from 150 to $250 \mathrm{~g}$ with the age range of 3-4 month. The rats were housed in sterile cages and kept at $25^{\circ} \dot{\mathrm{C}}$ with $12 / 12$ light-dark cycle. The rats allowed for chow pellet and to drink tap water ad libitum. Humane care for animals was according to the conduct to the care and utilize of laboratory animal under ethical endorsement permission.

After 2 weeks of acclimatization period, the animals were randomly divided into three groups, 10 rats in each group.

- Group I: (Negative control group) Received normal saline (5 ml/kg/day) for 10 days.

- Group II: (Positive control group) Received normal saline ( $5 \mathrm{ml} / \mathrm{kg} /$ day) for 10 days, and on the $8^{\text {th }}$ day, a single dose of doxorubicin (15 mg/kg, IP) (doxorubicin $\mathrm{HCl} 50 \mathrm{mg}$ Pfizer USA) 
soluble powder was given which serves as doxorubicin group.

- Group III: (Treated group) Received febuxostat (10 mg/kg, po) Feburic $120 \mathrm{mg}$ (Alhikma co. Jordon) daily for 10 successive days, and on the $8^{\text {th }}$ day, a $1 \mathrm{~h}$ after drug administration, a single dose of doxorubicin (15 mg/kg, I.P.) was given. The procedure of the study was according to Su et al. study [10].

At the $11^{\text {th }}$ day of the study, rats were sacrificed and hearts were taken for histopathological observations, blood samples were taken for biochemical analysis. Blood samples were collected through intracardiac puncture in sterile labeled tubes, then centrifuged for $10 \mathrm{~min}$ at $4500 \mathrm{rpm}$ and stored at $-20^{\circ} \mathrm{C}$ to be assessed later.

\section{Assessment of the biochemical variables}

Serum brain natriuretic peptide (BNP), cardiac troponin I (cTn-I), caspase-3, glutathione peroxidase (GSH-Px), lipid peroxidase (LPO), malondialdehyde (MDA), and tumor necrosis factor alpha (TNF- $\alpha$ ) were estimated by sandwich ELISA kit method (Kono Biotech Company, China).

\section{Assessment of the histopathological changes}

Fixation, tissue dissection, microtome sectioning, and slide preparation of rat hearts were according to Ashour et al. study [11].

\section{Statistics}

Data analysis was done using IBM SPSS (IBM SPSS Statistics for Windows version 20.0, 2014 Armonk, NY, IBM, Corp). The data were expressed as mean \pm standard deviation. The significance of the difference of different means (quantitative data) was tested using Student's t-test for difference between two independent means. Statistical significance was considered when $\mathrm{p}<0.05$.

\section{RESULTS}

Changes in the cardiac, inflammatory, oxidative, and apoptotic biomarkers in doxorubicin-induced acute cardiotoxicity

Results of the present study demonstrated that doxorubicin led to cardiotoxicity since it causes a significant elevation in the cardiac biomarkers. Cardiac troponin, BNP, caspase-3, and LPO were highly elevated compared to control $\mathrm{p}<0.01$, while MDA and TNF- $\alpha$ were elevated but to a lesser extent $\mathrm{p}<0.05$. In addition, GSH-Px serum levels were decreased significantly $\mathrm{p}<0.01$ compared to control group, Table 1.

Febuxostat administration before doxorubicin led to a significant decrease in cardiac troponin, caspase-3, and elevation in GSH-Px levels significantly $\mathrm{p}<0.05$. While the effect of febuxostat on BNP, LPO, MDA, tumor necrosis-alpha were insignificant $P>0.05$ compare to doxorubicin group Table 2 .

Histopathological changes in doxorubicin-induced acute cardiotoxicity

The control group section showed normal structure of myocardial tissue with peripherally located normal oval nucleus and branching striated muscle fibers. While, the section of doxorubicin group showed many congested and dilated blood vessels with extravasations, edema, cytoplasmic vaculations, decreased number of nuclei, and loss of muscle fibers striation. Regarding the effect of febuxostat, it led to the preservation of nuclei without muscles fibers fragmentation, but congested and dilated blood vessels with edema and extravasations are seen, Fig. 1 .

\section{DISCUSSION}

The present study clearly showed that doxorubicin induces myocardial injury by a significant elevation in plasma level of cTn-I in doxorubicintreated rats compared to the control. These findings are in agreement with the experimental research that showed the response of CTn-I to chemotherapy-induced acute myocardial injury [12].
It is well-known that cardiac troponin is regarded as the gold standard biomarker for myocardial injury and cardiotoxicity. It is only released into the plasma when cardiac myocytes were injured, so an increase in its level during treatment with doxorubicin reflects the acute cardiotoxicity of this chemotherapeutic agent. Thus, an elevation of CTn-I after a high dose of chemotherapy accurately predicts the severity of cardiac dysfunction [13].

Table 1: Serum level of cardiac biomarkers during doxorubicin-induced cardiotoxicity in rats compared to the control

\begin{tabular}{lccc}
\hline Parameters & $\begin{array}{c}\text { Control } \\
(\mathbf{n = 1 0})\end{array}$ & $\begin{array}{c}\text { Doxorubicin } \\
(\mathbf{n = 1 0 )}\end{array}$ & $\mathbf{p}$ \\
\hline BNP $(\mu \mathrm{g} / \mathrm{L})$ & $10.67 \pm 1.63$ & $17.17+1.94$ & $0.0001^{*}$ \\
Caspase-3 (pmol/L) & $13.33 \pm 2.42$ & $24.67 \pm 4.59$ & $0.0003^{*}$ \\
Cardiac troponin $(\mathrm{ng} / \mathrm{L})$ & $17 \pm 3.41$ & $42.0 \pm 7.54$ & $0.0001^{*}$ \\
GSH (pmol/L) & $24.83 \pm 3.97$ & $14.5 \pm 4.32$ & $0.001^{*}$ \\
LPO (nmol/L) & $14.83 \pm 1.72$ & $26.17 \pm 7.83$ & $0.006^{*}$ \\
MDA (nmol/L) & $1.1 \pm 0.414$ & $1.933 \pm 0.74$ & 0.03 \\
TNF (ng/L) & $23.17 \pm 11.09$ & $37.33 \pm 8.96$ & 0.03 \\
\hline
\end{tabular}

Results are expressed as mean $\pm S D ;{ }^{*}<<0.01, p<0.05$, BNP: Brain natriuretic peptide, GSH: Glutathione peroxidase, LPO: Lipid peroxidase, MDA: Malondialdehyde, TNF: Tumor necrosis factor alpha

Table 2: Effects of febuxostat on cardiac biomarker levels during doxorubicin-induced cardiotoxicity in rats

\begin{tabular}{lccc}
\hline Parameters & $\begin{array}{c}\text { Doxorubicin } \\
(\mathbf{n = 1 0})\end{array}$ & $\begin{array}{c}\text { Febuxostat } \\
\mathbf{( n = 1 0 )}\end{array}$ & $\mathbf{p}$ \\
\hline BNP $(\mu \mathrm{g} / \mathrm{L})$ & $17.17+1.94$ & $15.8 \pm 1.788$ & 0.25 \\
Caspase-3 $(\mathrm{pmol} / \mathrm{L})$ & $24.67 \pm 4.59$ & $20.4 \pm 1.14$ & $0.03^{*}$ \\
Cardiac troponin $(\mathrm{ng} / \mathrm{L})$ & $42.0 \pm 7.54$ & $32.8 \pm 4.21$ & $0.03^{*}$ \\
GSH $(\mathrm{pmol} / \mathrm{L})$ & $14.5 \pm 4.32$ & $19.2 \pm 3.34$ & $0.02^{*}$ \\
LPO $(\mathrm{nmol} / \mathrm{L})$ & $26.17 \pm 7.83$ & $24.6 \pm 2.23$ & 0.40 \\
MDA (nmol/L) & $1.933 \pm 0.74$ & $1.60 \pm 0.15$ & 0.14 \\
TNF $(\mathrm{ng} / \mathrm{L})$ & $37.333 \pm 8.96$ & $34.0 \pm 3.83$ & 0.25 \\
\hline
\end{tabular}

Results are expressed as mean $\pm S D ;{ }^{*}<<0.05$, BNP: Brain natriuretic peptide, GSH: Glutathione peroxidase, LPO: Lipid peroxidase, MDA: Malondialdehyde, TNF: Tumor necrosis factor alpha

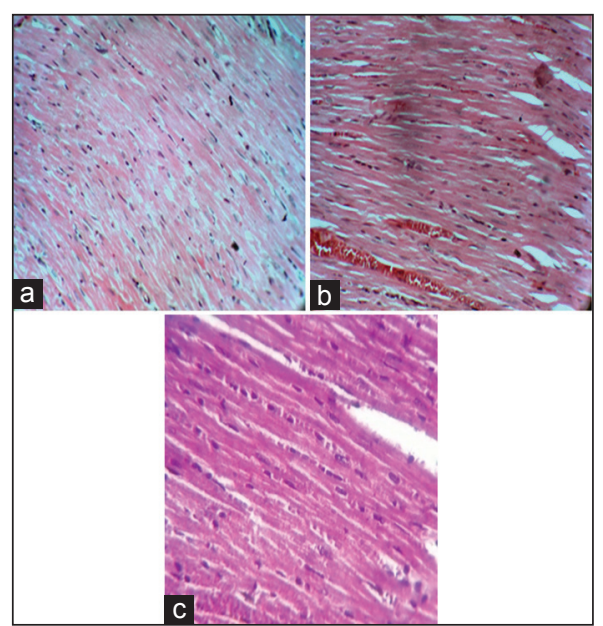

Fig. 1: Sections of myocardial tissue, magnification $\times 40$ ( $\mathrm{H}$ and $\mathrm{E}$ ). (a) (Control) Section showed normal rat myocardial tissue, (b) (doxorubicin) section showed congested and dilated blood vessel fragmented muscles fibers (orange arrow), decreased number of nuclei due to doxorubicin-induced acute cardiotoxicity, (c) (febuxostat) section showed preserved nuclei and no muscles fibers fragmentation but congested and dilated blood vessels with edema and extravasations due cardioprotective effect of febuxostat 
Therefore, the results of the present study pointed out that rats which were pre-treated with febuxostat showed reduction in CTn-I which is agreed with the previous study that has been described the response of cardiac troponins to DIC [14]

As well, the present study showed a significant increase in LPO in doxorubicin group compared to control group that corresponds with Khan et al. study that demonstrated a significant elevation in LPO levels in DIC [15]. Increase in LPO level could be attributed to the cardiomyocyte toxic effects of free radical oxidative degradation of doxorubicin [16]

Moreover, the results of the present study illustrated that rats which pre-treated with febuxostat showed significant decrease in LPO serum level compared to doxorubicin-treated rats, this finding is in agree with Wang et al. study that showed febuxostat therapy in animal model study causes a significant decline in serum levels of LPO due to inhibition of free radical generation [17].

Furthermore, MDA levels were significantly increased in the doxorubicin-treated rats compared to control group. This finding confirms the role of free radicals in DIC. Increase in MDA level could be attributed to the effects of free radicals that are generated as a result of doxorubicin effect, on NADH-dependent microsomal LPO that initiates lipid radical chain and oxidative damage $[18,19]$. Also, pre-treatment with febuxostat led to significant decrease in MDA serum level compared to doxorubicin treated rats which coincided with a study that demonstrated a significant decrease in MDA serum levels during febuxostat treatment [20].

Furthermore, TNF was significantly increased in doxorubicin group compared to the control group. Interestingly, both myocardial macrophages and cardiac myocytes synthesize TNF- $\alpha$. Accumulating evidence indicates that myocardial TNF- $\alpha$ is an autocrine contributor to myocardial dysfunction and cardiomyocyte death in ischemiareperfusion injury a sepsis and chronic heart failure [21].

Moreover, pre-treated with febuxostat showed insignificant decrease in serum TNF- $\alpha$ level compared to doxorubicin treated rats. This outcome is not corresponded with a study that disclosed a potential effect of febuxostat in reduction of pro-inflammatory cytokines [22].

In the present study, BNP was significantly increased in the doxorubicintreated rats compared to control group. In contrast, Ruggiero et al. reported low BNP serum levels during acute DIC due to inhibition of BNP gene expression [23]. However, in the present study, high levels of BNP were due to the development of acute heart failure as BNP serum levels are correlated with the severity of heart failure [24].

The cardioprotective effects of febuxostat might explain the reduction in BNP serum levels as febuxostat illustrates antioxidant and antiinflammatory effects as well as the reduction of serum uric acid which have deleterious effect on the cardiomyocyte [17].

Moreover, the present study showed that febuxostat decreases caspase-3 serum level compared to doxorubicin-treated rats. This results in agreement with the research that showed febuxostat reduced the apoptotic marker, namely caspase- 3 compared to doxorubicin-treated rats due to the antiapoptotic effect of febuxostat [25].

Regarding the effect on the endogenous antioxidant capacity, doxorubicin produced significant decreases of GSH-Px in DIC compared to the control group due to free radicals formation that attacks the glutathione. Febuxostat significantly improves body endogenous capacity through augmentation of antioxidant enzymes. On the other hand, febuxostat was ineffective in reduction of MDA serum levels and oxidative stress which might due to small dose of febuxostat in neutralization the effect of free radicals [26].
In the present study, structural changes that demonstrated microscopically in the heart of doxorubicin group compared to normal control group. Doxorubicin induced cardiac cell injury which revealed as congested and dilated blood vessels, extravasations, edema, cytoplasmic vaculations, decreased number of nuclei, loss of muscle fibers striation and fragmentation with necrosis [27].

In rats treated with febuxostat, the cardiac cells maintained their integrity by the evidence of preserved nuclei, the absence of fragmentation of the muscle fibers. It has been reported that febuxostat attenuated the cardiac cell injury by its antioxidant effect. Febuxostat limits the infarct size of acute myocardial infarction due to its antioxidant, anti-inflammatory, and antiapoptotic effect [28]

\section{CONCLUSION}

Febuxostat attenuates DIC through modulation of antioxidant, antiinflammatory, and antiapoptotic pathways.

\section{AUTHORS' CONTRIBUTIONS}

All authors contribute equally in data collection, experimental design, interpretation, statistical analysis, literature review, manuscript preparation, and review.

\section{CONFLICTS OF INTEREST}

Nil.

\section{REFERENCES}

1. Al-Kuraishy HM, Al-Gareeb AI. Potential effects of pomegranate on lipid peroxidation and pro-inflammatory changes in daunorubicininduced cardiotoxicity in rats. Int J Prev Med 2016;7:85.

2. Al-Kuraishy HM, Al-Gareeb AI. Interleukin-17: A cardiac biomarker in estimation of cardioprotective effects of tacrolimus in doxorubicininduced cardiotoxicity: Animal model study. Int J Recent Sci Res 2015;6:2769-74

3. Hayder MA, Al-Gareeb AI, Al-Hussaniy HA. Doxorubicin-induced cardiotoxicity: Molecular mechanism and protection by conventional drugs and natural products. Int J Clin Oncol Cancer Res 2017;2:31-44.

4. Ravichandra V, Hanumantharayappa B, Papasani VM. Evaluation of cardio protective activity of galangin against doxorubicin induced cardiomyopathy. Int J Pharm Pharm Sci 2014;6:86-90.

5. Kalyani C, Narasu ML, Devi Y. Synergistic growth inhibitory effect of flavonol-kaempferol and conventional chemotherapeutic drugs on cancer cells. Int J Pharm Pharm Sci 2016;9:123-7.

6. Durham KK, Chathely KM, Mak KC, Momen A, Thomas CT, Zhao YY, et al. HDL protects against doxorubicin-induced cardiotoxicity in a scavenger receptor class B Type 1-,PI3K-,and akt-dependent manner. Am J Physiol Heart Circ Physiol 2018;314:H31-44.

7. Kobayashi M, Usui F, Karasawa T, Kawashima A, Kimura H, Mizushina Y, et al. NLRP3 deficiency reduces macrophage interleukin-10 production and enhances the susceptibility to doxorubicin-induced cardiotoxicity. Sci Rep 2016;6:26489.

8. Sarvepalli PS, Fatima M, Quadri AK, Taher AR, Habeeb A, Amreen F, et al. Study of therapeutic efficacy of febuxostat in chronic kidney disease stage IIIA to stage VD. Saudi J Kidney Dis Transpl 2018;29:1050-6.

9. Krishnamurthy B, Rani N, Bharti S, Golechha M, Bhatia J, Nag TC, et al. Febuxostat ameliorates doxorubicin-induced cardiotoxicity in rats. Chem Biol Interact 2015;237:96-103.

10. Su S, Li Q, Liu Y, Xiong C, Li J, Zhang R, et al. Sesamin ameliorates doxorubicin-induced cardiotoxicity: Involvement of sirt1 and mn-SOD pathway. Toxicol Lett 2014;224:257-63.

11. Ashour OM, Elberry AA, Alahdal A, Al Mohamadi AM, Nagy AA, Abdel-Naim AB, et al. Protective effect of bilberry (Vaccinium myrtillus) against doxorubicin-induced oxidative cardiotoxicity in rats. Med Sci Monit 2011;17:BR110-5.

12. Reagan WJ, York M, Berridge B, Schultze E, Walker D, Pettit S, et al. Comparison of cardiac troponin I and $\mathrm{T}$, including the evaluation of an ultrasensitive assay, as indicators of doxorubicin-induced cardiotoxicity. Toxicol Pathol 2013;41:1146-58.

13. Dylan S, Chan SH, Yip S, Rodis B, Starovoytov A, Lam L, et al. clinical outcomes of acute ischemic stroke patients stratified by cardiac troponin-i: A retrospective analysis. J Am Coll Cardiol 2017;69:140 
14. Montenegro MF, Lundberg JO, Weitzberg E. Cardiovascular safety of febuxostat. N Engl J Med 2018;379:1583-4.

15. Khan G, Haque SE, Anwer T, Ahsan MN, Safhi MM, Alam MF, et al. Cardioprotective effect of green tea extract on doxorubicin-induced cardiotoxicity in rats. Acta Pol Pharm 2014;71:861-8.

16. Niki E. Biomarkers of lipid peroxidation in clinical material. Biochim Biophys Acta 2014;1840:809-17.

17. Wang S, Li Y, Song X, Wang X, Zhao C, Chen A, et al. Febuxostat pretreatment attenuates myocardial ischemia/reperfusion injury via mitochondrial apoptosis. J Transl Med 2015;13:209.

18. Al-Kuraishy HM, Al-Gareeb AI. Eustress and malondialdehyde (MDA): Role of panax ginseng: Randomized placebo controlled study. Iran J Psychiatry 2017; 12:194-200.

19. Al-Kuraishy HM, Al-Gareeb AI, Al-Maiahy TJ. Concept and connotation of oxidative stress in preeclampsia. J Lab Physicians 2018; 10:276-82.

20. Shafik AN. Febuxostat improves the local and remote organ changes induced by intestinal ischemia/reperfusion in rats. Dig Dis Sci 2013;58:650-9.

21. Zhao L, Zhang B. Doxorubicin induces cardiotoxicity through upregulation of death receptors mediated apoptosis in cardiomyocytes. Sci Rep 2017;7:44735
22. Ramos MF, Monteiro de Barros AD, Razvickas CV, Borges FT, Schor N. Xanthine oxidase inhibitors and sepsis. Int J Immunopathol Pharmacol 2018;32:2058738418772210.

23. Ruggiero A, De Rosa G, Rizzo D, Leo A, Maurizi P, De Nisco A, et al. Myocardial performance index and biochemical markers for early detection of doxorubicin-induced cardiotoxicity in children with acute lymphoblastic leukaemia. Int J Clin Oncol 2013;18:927-33.

24. Zelt JG, Liu PP, Erthal F, deKemp RA, Wells G, O'Meara E, et al. $\mathrm{N}$-terminal pro B-type natriuretic peptide and high-sensitivity cardiac troponin $\mathrm{T}$ levels are related to the extent of hibernating myocardium in patients with ischemic heart failure. Can J Cardiol 2017;33:1478-88.

25. Khan SI, Malhotra RK, Rani N, Sahu AK, Tomar A, Garg S, et al. Febuxostat modulates MAPK/NF- $\mathrm{KBp} 65 / \mathrm{TNF}-\alpha$ signaling in cardiac ischemia-reperfusion injury. Oxid Med Cell Longev 2017;2017:8095825.

26. Kapoor N, Saxena S. Xanthine oxidase inhibitory and antioxidant potential of Indian muscodor species. 3 Biotech 2016;6:248.

27. Al-Kurishy $\mathrm{H}, \mathrm{Al}-\mathrm{Gareeb}$ AI. Advances and prospects of nicardipine effects in attenuation ofhydroxy-daunorubicin induced acute cardiotoxicity in rats. Adv Biomed Pharm 2015;2:274-82.

28. White WB, Chohan S, Dabholkar A, Hunt B, Jackson R. Cardiovascular safety of febuxostat and allopurinol in patients with gout and cardiovascular comorbidities. Am Heart J 2012;164:14-20. 